\title{
Molecular Characterization and Expression Profiling of Nuclear Receptor Gene Families in Oriental Fruit Fly, Bactrocera Dorsalis (Hendel)
}

\author{
Pei-Jin Yang ${ }^{1,2}$, Er-Hu Chen ${ }^{1,2}$, Zhong-Hao Song ${ }^{1,2}$, Wang He ${ }^{1,2}$, Shi-Huo Liu ${ }^{1,2} \mathbb{D}$, \\ Wei Dou ${ }^{1,2, *}$ and Jin-Jun Wang ${ }^{1,2}$ (D) \\ 1 Key Laboratory of Entomology and Pest Control Engineering, College of Plant Protection, Southwest \\ University, Chongqing 400715, China; y505302304@gmail.com (P.-J.Y.); erhuchen1104@yahoo.com (E.-H.C.); \\ pathetiquesong@163.com (Z.-H.S.); wangheswu@163.com (W.H.); liushihuo@email.swu.edu.cn (S.-H.L.); \\ wangjinjun@swu.edu.cn (J.-J.W.) \\ 2 Academy of Agricultural Sciences, Southwest University, Chongqing 400715, China \\ * Correspondence: douwei80@swu.edu.cn
}

Received: 17 December 2019; Accepted: 14 February 2020; Published: 16 February 2020

\begin{abstract}
The oriental fruit fly (Bactrocera dorsalis) is a pest that causes large economic losses in the fruit and vegetable industry, so its control is a major challenge. Nuclear receptors (NRs) are a superfamily of ligand-dependent transcription factors that directly combine with DNA to regulate the expression of downstream target genes. NRs are closely associated with multiple physiological processes such as metabolism, reproduction, and development. Through sequence searches and analysis, we identified $21 \mathrm{~B}$. dorsalis NR genes, all of which contained at least one of the two characteristic binding domains. On the basis of the conserved sequences and phylogenetic relationships, we divided the 21 NR genes into seven subfamilies. All members of the NR0 subfamily and $B d H R 83$, which belonged to the NR2E group, lacked ligand-binding domains. The BdDSF and $B d H R 51$, which also belonged to the NR2Egroup, and BdE78 (which belonged to the NR1E group) all lacked DNA-binding domains. The BdDSF and BdHR83 sequences were incomplete, and were not successfully amplified. Development- and tissue-specific expression profiling demonstrated that the transcript levels of the 19 NR genes varied considerably among eggs, larva, pupae, and adults, as well as among larval and adult male and female tissues. Our results will contribute to a better understanding of NR evolution and expand our knowledge of $B$. dorsalis physiology.
\end{abstract}

Keywords: Bactrocera dorsalis; transcription factors; spatiotemporal expression; development

\section{Introduction}

Nuclear receptors (NRs) are a large family of transcriptional regulators. They are involved in a variety of physiological functions, including controlling embryonic development, regulating cell differentiation, and maintaining homeostasis. NRs and cofactors on target genes interact with the response sequence in the form of monomers, homodimers and heterodimers [1]. In general, NRs bind receptor ligands for transcriptional regulation, and receptor ligands are usually composed of small lipid molecules, such as retinoids or steroids. However, the regulatory ligands for some receptors (orphan receptors) are still unknown or may not exist [2].

NRs are evolutionarily conserved proteins that have been divided into seven distinct subfamilies and contain a characteristic modular structure [3]. The A/B domains contain a transcriptional activation function (AF-1) and are highly variable with very little evolutionary conservation. The $C$ or DNA-binding domain (DBD) is the most highly conserved domain. It contains two typical cysteine-rich zinc finger motifs in tandem that span about 80 amino acids and are involved directly 
in recognizing hormone response elements. The $\mathrm{D}$ domain functions as a hinge between the DBD and the ligand-binding domain (LBD). The LBD (also known as E domain) contains a hydrophobic ligand-binding pocket that is specific to each receptor and is the source of the sequence variability within the LBD. In addition, the LBD domain mediates dimerization and the ligand-dependent transcriptional activation function (AF-2) [4]. The C-terminal contains the F domain, which is not present in all NRs and is highly diverse.

A large number of NR gene sets have been characterized from the whole-genome sequence of many animals. For example, 48 NRs were identified in the human genome [5], over 270 NRs have been found in Caenorhabditis elegans [6], and 49 and 47 were found in mouse and rat, respectively [7]. Surprisingly, only 21 NRs were found in Drosophila melanogaster [8], 19 in Bombyx mori [9], 22 in Apis mellifera [10], and 21 in Tribolium castaneum [11].

In D. melanogaster, seven NR genes that are regulated directly by 20E have been identified, namely EcR, E75, E78, FTZ-F1, HR3, HR4, and HR39 [12]. Among them, E75 is the primary response gene of 20E. The others are secondary response genes that are maximally expressed after 20E-induced protein synthesis has begun [13]. The ligand-receptor complex of 20E-EcR-USP induces the expression of the $20 \mathrm{E}$ primary response gene. Then, the cascade of $20 \mathrm{E}$ secondary response genes, which is induced by the transcription factor encoded by the 20E primary response gene, amplifies the 20E signal by regulating the expression of the secondary response genes (e.g., HR3, HR4, and FTZ-F1), thus regulating the physiological processes of molting, metamorphosis, and reproduction in insects [14]. Precise regulation of 20E requires not only 20E-dependent NRs, but also NRs that inhibit its transcriptional regulation (e.g., HR78, HR38, and seven-up (SVP)). These NRs weaken the activity of the 20E signaling pathway by inhibiting the normal function of EcR (Ecdysone Receptor) /USP (Ultraspiracle) dimers. Ten NR genes in D. melanogaster are not dependent on 20E. They play a key role in the physiological processes related mainly to embryo formation, neurodevelopment, metabolism, and detoxification. For example, the nuclear hormone receptor HR96 mediates resistance to foreign compounds [15] and HR78 plays a major role in promoting the expression of genes in the midgut, suggesting it may contribute to nutrient uptake [16].

The oriental fruit fly (Bactrocera dorsalis) is a devastating and adaptable insect (Diptera: Tephritidae) with a wide range of food sources that include 450 different kinds of fruits and vegetables. This pest poses a serious threat to the fruit and vegetable industry and can cause very serious economic losses [17]. The aim of this study was to identify and annotate NRs in the B. dorsalis genome, analyze their structural domains, and construct an evolutionary tree. We inferred gene expression by quantitative real-time PCR (qRT-PCR) to validate the key genes that control development and explore the regulatory mechanism of NR genes in B. dorsalis.

\section{Materials and Methods}

\subsection{Insect Cultures}

The B. dorsalis laboratory population was collected from Hainan Province, China, in 2008. The insects were kept at $27.5 \pm 0.5{ }^{\circ} \mathrm{C}$ with $75 \pm 5 \%$ humidity and a photoperiod of 14:10 h (L:D). The larval feed was composed mainly of agar, yeast, sugar, wheat flour, and corn flour. The adult feed was composed mainly of yeast, sugar, honey, and water. The specific proportions of each ingredient in these artificial diets were as described previously [18] .

\subsection{Total RNA Extraction and cDNA Synthesis}

Total RNA of each sample was extracted using TRIzol ${ }^{\circledR}$ reagent (Invitrogen, Carlsbad, CA, USA). The quality and concentration of RNA were both measured by NanoVue UV-Vis spectrophotometer (GE Healthcare Bio-Sciences, Uppsala, Sweden), and the integrity of isolated RNA was checked by $1.0 \%$ agarose gel electrophoresis. Prior to cDNA synthesis, total RNA was treated with RQ1 RNase-Free DNase (Promega, Madison, WI, USA) to digest genomic DNA. For first strand cDNA 
synthesis, $500 \mathrm{ng}$ of total RNA was reverse transcribed using the PrimeScript 1st Strand cDNA Synthesis Kit (Takara, Dalian, China) with random hexamers and oligo (dT) primers, according to the manufacturer's instructions.

\subsection{Identification and Annotation of NRs}

To identify and characterize the NR-related proteins of $B$. dorsalis, we downloaded NR amino acid sequences of D. melanogaster and B. dorsalis from FlyBase (http://flybase.org/) and GenBank (http://www. ncbi.nlm.nih.gov). The NR sequences were used as queries in tBLASTn searches to identify homologous and related genes in the $B$. dorsalis genome database (i5K; https://i5k.nal.usda.gov/webapp/blast/). All the thresholds were set to default and we only took the top hit result. The specific Drosophila melanogaster sequences we used as queries are listed in Table S1. Before the BLAST searches, we had confirmed that all the NRs in Drosophila melanogaster were unique. In the subsequent BLAST seearches, the highest result of hits was only one, and there were no multiple results that met the requirements. Furthermore, we used the best reciprocal hits of BLAST to check the NRs in B. dorsalis and no false positives were recorded. This indicated that no NR duplication events existed in B. dorsalis.

\subsection{Phylogenetic Analysis}

To understand the relationship of $B$. dorsalis NRs (BdNRs) with those of a model dipteran species and other closely related species, the NR amino acid sequences from $B$. dorsalis, D. melanogaster, Bombyx mori, T. castaneum, and Ceratitis capitata downloaded from the NCBI (National Center for Biotechnology Information) database (https:/www.ncbi.nlm.nih.gov/), FlyBase, BeetleBase and the Silkworm Genome Database (http://silkworm.genomics.org.cn/) were used to construct a phylogenetic tree using MEGA 7.0 (Kuma et al., 2016). Subsequently, neighbor-joining trees were built by applying the Poisson correction model and pairwise deletion method for gaps. Bootstrap iterations to assess the robustness of the generated trees were set to 1000 repetitions. The sequences of 90 NRs from five insect species for the phylogenetic analysis are listed in Table S2.

\subsection{Domain Analysis}

The DBDs and LBDs were identified in the NR protein sequences of $B$. dorsalis using SMART (http://smart.embl-heidelberg.de/) and the Conserved Domains Search (https://www.ncbi.nlm.nih.gov/ Structure/cdd/wrpsb.cgi). We used the ExPASy Compute pI/MW tool (http://cn.expasy.org/tools/pi_ tool.html) to obtain the theoretical pI (isoelectric point) and molecular weight (MW) of the deduced NR proteins. We chose NR0, NR1 and NR2 three subfamilies from B. dorsalis and D. melanogaster and used DNAMAN 6 (https://www.lynnon.com/downloads.html) for the multiple sequence alignment. All parameters were set to default.

\subsection{Temporal and Developmental Expression Profiles}

Samples were collected at different development stages (eggs; one-, three-, five-, seven- and nine-day-old larvae; one-, three-, five-, seven- and nine-day-old pupae; and one-, three-, five-, sevenand nine-day-old male and female adults) and from different tissues (midgut, fat body, integument, Malpighian tubule, and central nervous system of third instar larva; and midgut, fat body, Malpighian tubule, ovary, and testis of four-day-old adults). Specific primers for quantitative real-time PCRs (qRT-PCRs) were designed using Primer 3 web (http://primer3.ut.ee/) and synthesized by Invitrogen in Shanghai, China. The qRT-PCRs were conducted in a $10-\mu \mathrm{L}$ reaction mixture that included $5 \mu \mathrm{L}$ qPCR Master Mix (Promega, Madison, WI), $0.5 \mu \mathrm{L}$ cDNA templates (about $400 \mathrm{ng} / \mu \mathrm{L}$ ), and $0.5 \mathrm{mM}$ each of forward and reverse primers. The PCR cycles were $95^{\circ} \mathrm{C}$ for $2 \mathrm{~min}$, then 35 cycles at $95{ }^{\circ} \mathrm{C}$ for $15 \mathrm{~s}$, $55^{\circ} \mathrm{C}$ for $30 \mathrm{~s}$ and $72{ }^{\circ} \mathrm{C}$ for $20 \mathrm{~s}$, with a final extension at $72{ }^{\circ} \mathrm{C}$ for $7 \mathrm{~min}$. A single PCR product was verified by melting curve analysis from 65 to $95^{\circ} \mathrm{C}$. Three pairs of primers were used in BdDSF and $B d H R 83$, but the amplification failed. All primers used for reverse transcription PCR were listed in Table S3. Three to four independent biological replicates and two technical replicates were performed 
for each qRT-PCR. Each replication contained at least two individuals for the developmental stage pattern. Eggs and specific tissues were collected and dissected from 20 individuals to obtain enough RNA. The relative expression levels were obtained using the $\Delta C_{T}$ method with $\alpha$-tubulin and $r p s 3$ as the reference genes. We confirmed the presence of a single PCR product at the anticipated size by single melting curve and analyzed changes in the transcript levels of the NR genes using SPSS 16.0 (SPSS Inc., Chicago, IL, USA). The spatiotemporal expression level differences were determined by one-way analysis of variance (ANOVA), followed by Tukey's test at a significance level of 0.05 .

\section{Results}

\subsection{NR Identification and Characterization}

We identified 21 NR genes in the B. dorsalis genome. The gene names, GenBank accession numbers, pI and MW parameters, and corresponding NRs in D. melanogaster are listed in Table 1. The BdHR83 and BdDSF sequences were incomplete, which made subsequent quantitative analysis unreliable. The BdNRs were divided into seven subfamilies. The atypical NRs that contained only one conserved domain (DBD or LBD) were independently classified regardless of the degree of evolutionary correlation. Each subfamily was divided into several groups. The DBD homology among members of the same group was $80 \%-90 \%$ and the LBD homology was $40 \%-60 \%$. Three members of the NR0 subfamily (BdKNRL, BdKNI, and BdEGON) contained only DBDs (Figure 1). The NR1 subfamily contained five members, $B d E 75, B d E 78, B d E c R, B d H R 3$, and $B d H R 96$. The NR2 subfamily contained eight members, most of which belonged to the NR2E group. In addition, the rest contained one NR3, one NR4, two NR5 and one NR6 subfamily members (Table 1).
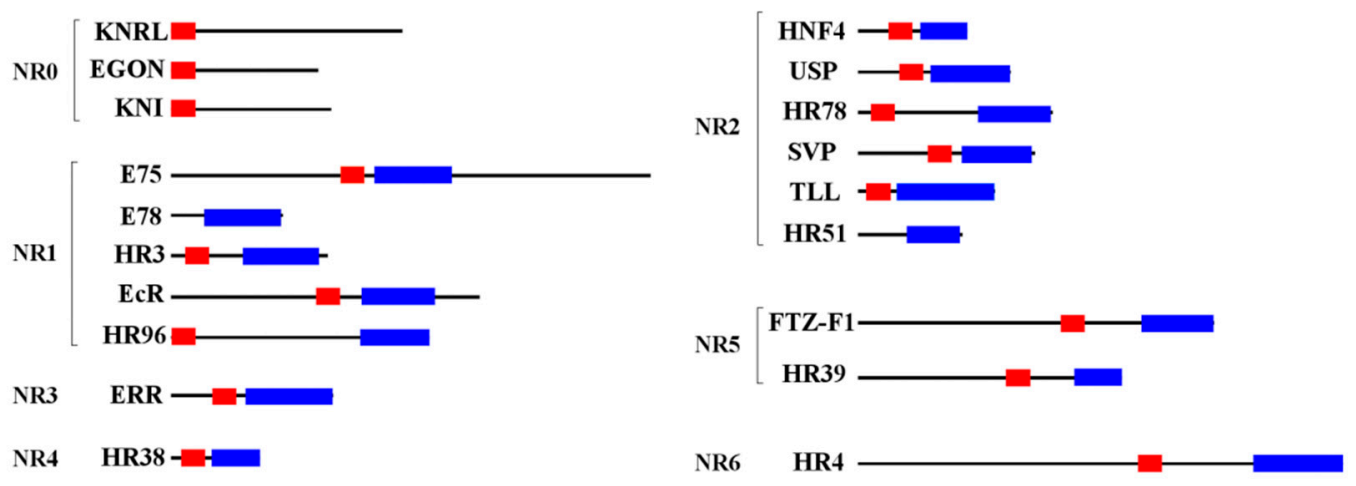

DBD: - LBD:

Figure 1. Domain architectures of 21 NR proteins in B. dorsalis. DNA-binding domain (DBD) (red); ligand-binding domain (LBD) (blue). The deduced amino acid sequences were used to predict the domain architectures in SMART (http://smart.embl-heidelberg.de/) and NCBI Conserved Domains Search (https://www.ncbi.nlm.nih.gov/Structure/cdd/wrpsb.cgi).

Table 1. List of the NR proteins identified in the B. dorsalis genome and NRs in D. melanogaster.

\begin{tabular}{ccccccc}
\hline Group & B. dorsalis & $\begin{array}{c}\text { Genbank } \\
\text { Accession no. }\end{array}$ & Protein Length/aa & M.W./kDa & pI & D. melanogaster \\
\hline OA & BdKNRL & XP_019844421 & 750 & 79.4 & 6.4 & KNRL \\
0A & BdEGON & XP_011213892 & 477 & 50.2 & 9.2 & EGON \\
OA & BdKNI & XP_029404449 & 519 & 54.5 & 6.2 & KNI \\
\hline
\end{tabular}


Table 1. Cont.

\begin{tabular}{ccccccc}
\hline Group & B. dorsalis & $\begin{array}{c}\text { Genbank } \\
\text { Accession no. }\end{array}$ & Protein Length/aa & M.W./kDa & pI & D. melanogaster \\
\hline 1D & BdE75 & XP_019846264 & 1302 & 144.7 & 8.2 & E75 \\
1E & BdE78 & XP_019846486 & 363 & 38.8 & 5.2 & E78 \\
1F & BdHR3 & XP_011208441 & 485 & 54.8 & 6.1 & HR3 \\
1H & BdECR & XP_011203444 & 566 & 62.8 & 6.9 & ECR \\
1J & BdHR96 & XP_019847193 & 847 & 94.8 & 5.7 & HR96 \\
2A & BdHNF4 & XP_029404261 & 698 & 76.8 & 6.4 & HNF4 \\
2B & BdUSP & XP_019845796 & 453 & 51.3 & 8.5 & USP \\
2D & BdHR78 & XP_011201915 & 630 & 69.2 & 5.5 & HR78 \\
2E & BdTLL & XP_011214380 & 516 & 57.2 & 5.6 & TLL \\
2E & BdDSF & XP_011214839 & 603 & 64.8 & 7.1 & DSF \\
2E & BdHR83 & XP_029407834 & 318 & 35.7 & 9.0 & HR83 \\
2E & BdHR51 & XP_011200189 & 339 & 37.4 & 5.0 & HR51 \\
2F & BdSVP & XP_029407513 & 574 & 60.8 & 8.6 & SVP \\
3B & BdERR & XP_019846473 & 524 & 57.4 & 7.0 & ERR \\
4A & BdHR38 & XP_011212592 & 822 & 93.1 & 9.0 & HR38 \\
5A & BdFTZ-F1 & XP_029405285 & 751 & 80.9 & 6.8 & FTZ-F1 \\
5B & BdHR39 & XP_019844854 & 856 & 89.4 & 7.3 & HR39 \\
6A & BdHR4 & XP_019846713 & 1573 & 169.1 & 9.1 & HR4 \\
\hline
\end{tabular}

(a) Molecular weight (MW) and isoelectric point (pI) were predicted using the ExPASy ProtParam tool available at http://web.expasy.org/protparam/. (b) Full-length cDNA sequences were not obtained in this study; characteristic parameters were predicted based on the NCBI Reference Sequences.

Most of the NR amino acid sequences were 300-900 amino acids in length, had MWs of 35.7-94.8 $\mathrm{kDa}$, and pIs from 5.0-9.2. BdE75 (1302 aa; $144.7 \mathrm{kDa}$ ) and BdHR4 (1573 aa; $169.1 \mathrm{kDa}$ ) were exceptions with longer sequences and higher MWs. Most of the NRs had DBDs and LBDs in their sequences; the exceptions were the NRs in the NR0 subfamily and BdHR83, which only had DBDs, and BdE78, BdDSF, and BdHR51, which only had LBDs.

\subsection{Phylogenetic and Structural Analysis}

The phylogenetic tree constructed using the 19 NRs of $B$. dorsalis (excluding BdHR83 and BdDSF) and the available NRs from B. mori, C. capitata, D. melanogaster, and T. castaneum (Figure 2) showed that the NRs did not cluster in species-specific clades, rather they segregated into the different subfamilies. Most of the NRs were highly similar to the D. melanogaster NRs (DmNRs), and the same subfamily proteins clustered into the same branches. Each subfamily represented NRs with distinct functions; for example, one cluster contained NRs that were related to growth and development. 


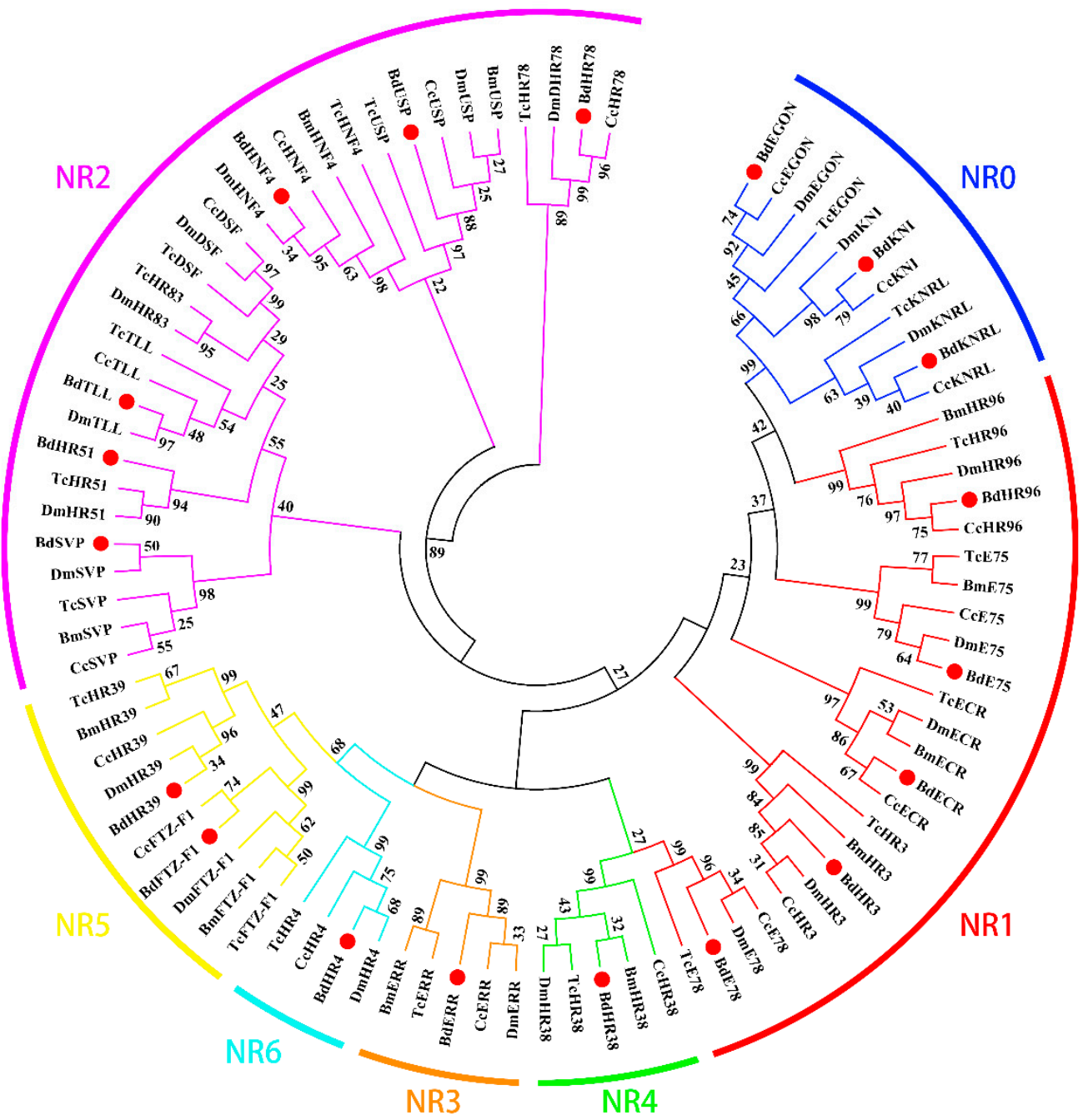

Figure 2. Phylogenetic analysis of 90 insect nuclear receptor (NR) proteins from five different species. Bombyx mori $(\mathrm{Bm})$, Ceratitis capitata $(\mathrm{Cc})$, Bactrocera dorsalis $(\mathrm{Bd})$, Drosophila melanogaster $(\mathrm{Dm})$ and Tribolium castaneum (Tc). A bootstrap analysis of 1000 replications was carried out on the trees inferred using the neighbor-joining method, and bootstrap values were shown at each branch of the tree. NRs from $B$. dorsalis are indicated by red dots.

\subsection{Spatiotemporal Expression Patterns}

Developmental stage and tissue-specific expression of the B. dorsalis NRs determined by qRT-PCR, are displayed as heatmaps (Figures $3-5$ ). The 19 BdNR genes were expressed in all stages from eggs to adult. $B d E 75, B d H R 4$, and $B d H R 3$ were highly expressed in five- and seven-day-old pupae; $B d S V P$ and $B d U S P$ expression levels were lowest in eggs and three-day-old adult females, respectively; BdTLL was highly expressed in eggs and five- to seven-day-old adult females; and the relative expression levels of $B d K N I$ and $B d K N R L$ were stable from eggs to adult (Figure 3). The raw expression data were listed in Table S4.

Among the different adult tissues, $B d T L L$ and $B d H R 3$ were highly expressed in the ovary, whereas the base expression of $B d T L L$ was very low in the female Malpighian tubule, male midgut, and fat body (Figure 4). In the larval tissues, BdTLL, BdHR38, and BdHR51 were highly expressed in the central nervous system (Figure 5). The raw expression data were listed in Tables S5 and S6. 


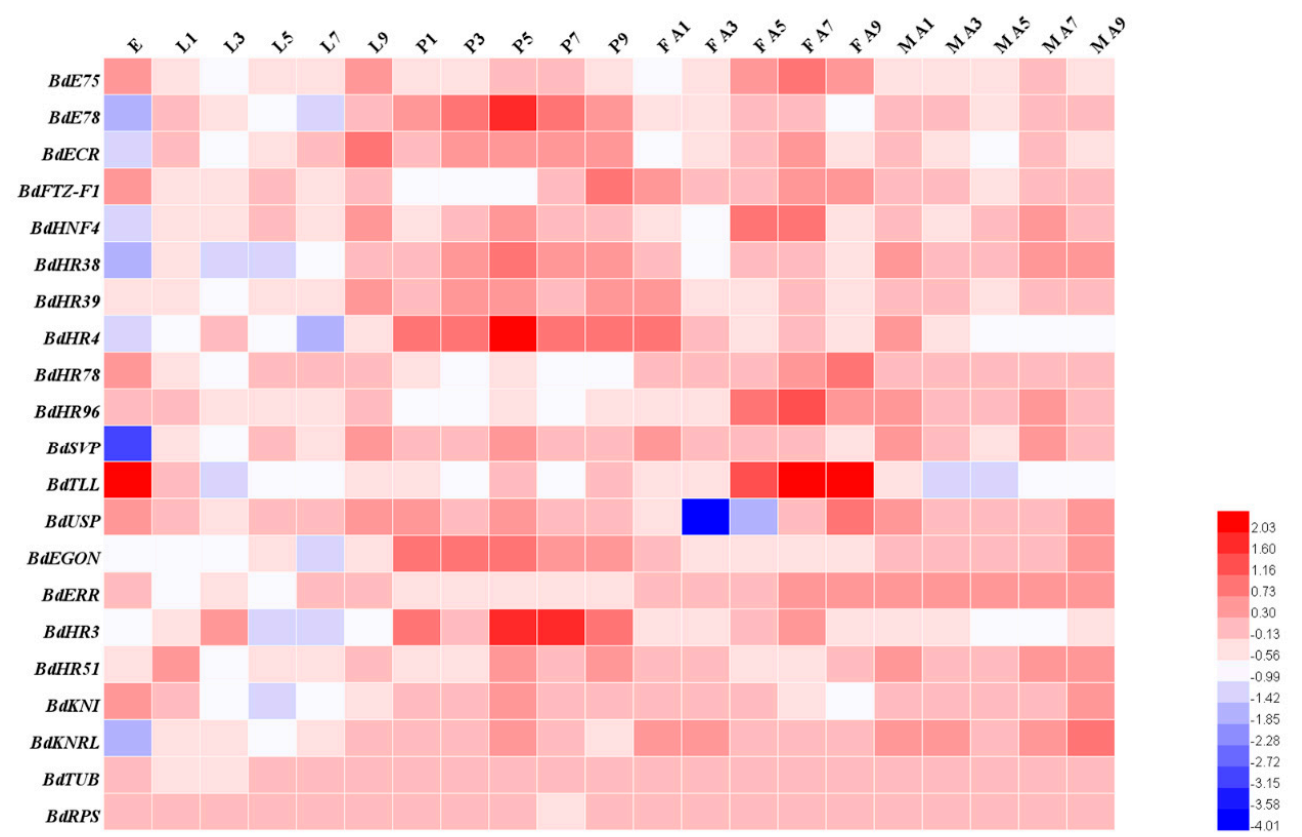

Figure 3. Developmental mRNA expression profiles of 19 B. dorsalis NR genes (excluding BdHR83 and $B d D S F)$. Relative transcript levels were calculated using $\alpha$-tubulin (GenBank Accession no.: GU269902) and rps3 (GenBank Accession no.: XM_011212815) as internal references. Three to four independent biological replicates and two technical replicates were performed for each qRT-PCR. E: eggs; L1, L3, L5, L7, and L9, one- to nine-day-old larva on odd days; P1, P3, P5, P7, and P9, one- to nine-day-old pupae on odd days; F: A1, A3, A5, A7, and A9, one- to nine-day-old female adults on odd days; M A1, A3, A5, A7, A9, one- to nine-day-old male adults on odd days. Warm colors (i.e., red) mean high expression levels and cold colors (i.e., blue) mean low expression levels.

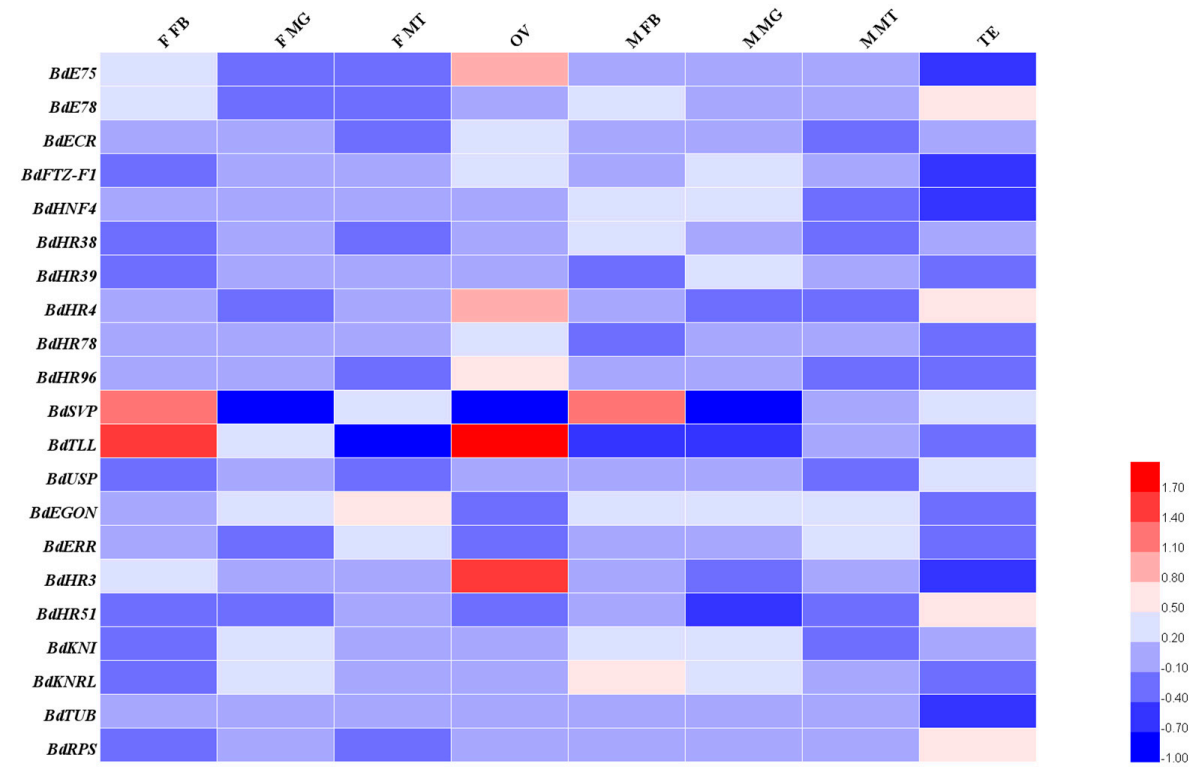

Figure 4. Tissue-specific expression of 19 B. dorsalis NR genes (excluding BdHR83 and BdDSF) from four-day-old adults. Relative transcript levels were calculated using $\alpha$-tubulin (GenBank Accession no.: GU269902) and rps3 (GenBank Accession no.: XM_011212815) as internal references. Three to four independent biological replicates and two technical replicates were performed for each qRT-PCR. Midgut of female/male adults (F/M MG); fat body of female/male adults (F/M FB); Malpighian tubule of female/male adults (F/M MT); ovary of adult females (OV); testis of adult males (TE). Warm colors (i.e., red) indicate high expression levels and cold colors (i.e., blue) indicate low expression levels. 


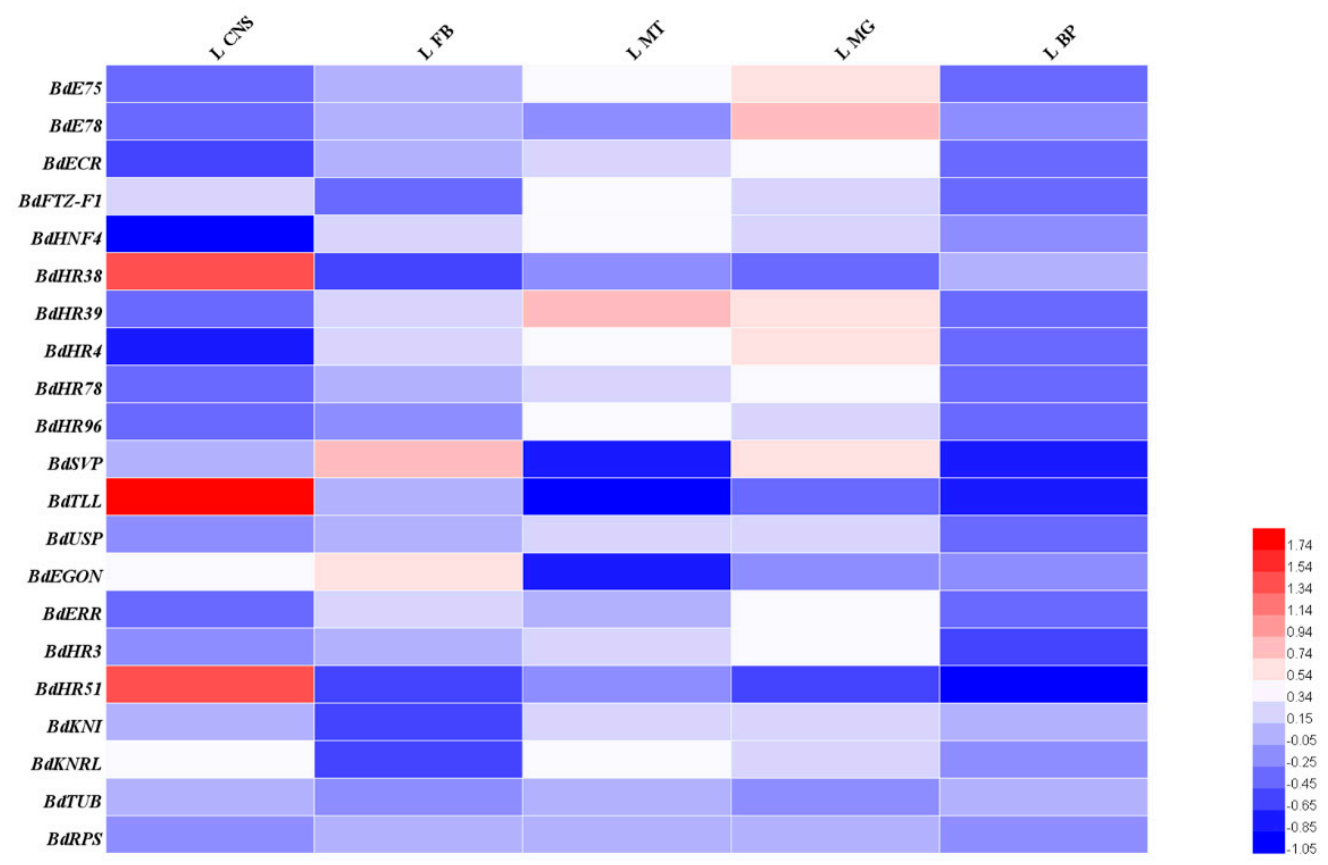

Figure 5. Tissue-specific expression of 19 NR B. dorsalis genes (excluding BdHR83 and BdDSF) from third instar larvae. Relative transcript levels were calculated using $\alpha$-tubulin (GenBank Accession no.: GU269902) and rps3 (GenBank Accession no.: XM_011212815) as internal references. Three to four independent biological replicates and two technical replicates were performed for each qRT-PCR. Larval midgut (L MG); larval fat body (L FB); larval cuticle (L BP); larval Malpighian tubule (L MT); larval central nervous system (L CNS). Warm colors (i.e., red) indicate high expression levels and cold colors (i.e., blue) indicate low expression levels.

\subsection{Multisequence Alignment}

Conserved regions in the NR0, NR1 and NR2 subfamily of $B$. dorsalis and D. melanogaster NR proteins were presented in Figures 6-8. The B. dorsalis genome contained three members of the NR0 subfamily. The $B d K N R L, B d K N I$, and BdEGON receptors were similar to the D. melanogaster Knirp receptors DmKNRL, DmKNI, and DmEGON and shared a high identity at their 5' ends (Figure 6), whereas there was very little similarity at their $3^{\prime}$ ends. The $B$. dorsalis genome contained five members of the NR1 subfamily. These BdNRs were similar to DmNRs (Table 1, Figure 1). They phylogenetically segregated into the NR1 clade and displayed few similarities to other NR groups (Figure 7). The $B$. dorsalis genome contained eight members of the NR2 subfamily. These BdNRs were similar to those of D. melanogaster; however, the BdHR83, BdHR51, and BdDSF sequences were incomplete, and only BdHR51 was successfully amplified (Table 1, Figure 1). The remaining five members all had high homology with members of the D. melanogaster NR2 subfamily; they shared high identity at the $5^{\prime}$ ends but had little similarity at the 3 ' ends (Figure 8 ). 


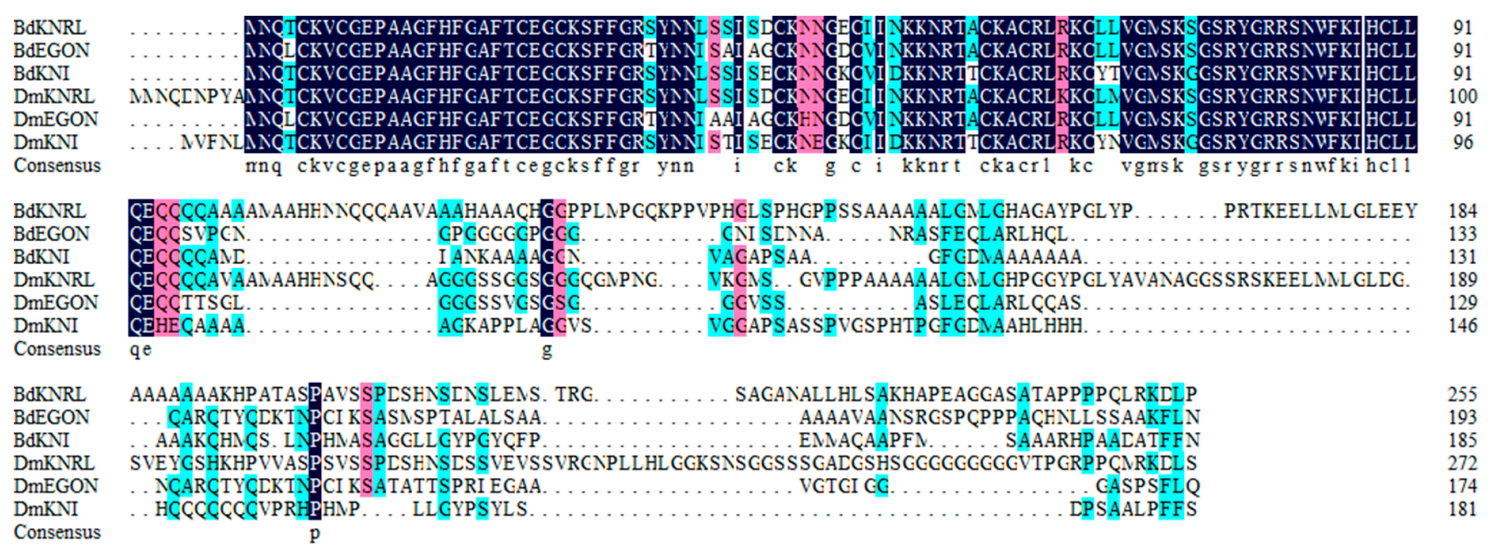

Figure 6. Conserved regions in the NR0 subfamily of B. dorsalis and D. melanogaster NR proteins. Amino acid sequences of the NR0 subfamily catalytic domains were aligned using DNAMAN 6 (https://www.lynnon.com/downloads.html). Identical and highly conserved amino acids are indicated by black $(100 \%)$, pink $(75 \%)$, and blue $(50 \%)$, respectively.

\begin{tabular}{|c|c|}
\hline $\begin{array}{l}\text { BdE75 } \\
\text { BdE78 }\end{array}$ & 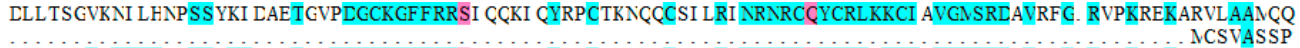 \\
\hline BdHR3 & CEGCKGFFRRSCSSVVNYC CPRNKCCVVDR NRNRCCYCRL CKCL CL GNSRLAVKFG RNSKKCREKVEEEVKF \\
\hline BdECR & RLCEELCLVCGLRASGYHYNALT. CEGCKGFFRRSVTKNAVYC. CKF GRSCENLNYMRRKCCECRNKKCL AVGNRPECVVPE. NCCANKRREKKACKEK \\
\hline BdHR96 & SNLNNSPVN THLFVNGVSPRECRSNSVTNSLI NVESPI NI CQGNI RTLTPLHTL TPI CQSCTLCLGSS AELPSVTACNENSTCGI SI TPTLCSI LNHALHC \\
\hline DmE75 & LGTTVLCRVCGLKASGFHYGVHS. . CEGCKGF FRSI CCKI CYRPCTKNCCCSI LRI NRNRCCYCRLKKCI AVGISRLAVRFG. RVPKREKARI LAANCQ \\
\hline DmE78 & CEGCKGFF RRSI CKCI EYR. CLRLGKCL VI RL NRNRCCYCRFKKCL SAGNSRDSVRYG. RVPKRSREL NGAAASS \\
\hline DmHR3 & CEGCKGF FRRSCSSVVNYC. CPRNKCCVVDR NRNRCCYCRL CKCL KL GISRDAVKFG. RLSKKQREKVELEVRF \\
\hline DMECR & CEGCKGF FRR STKS AVYC. CKF GRACENDN YMRRKCQECRLKKCL AVGNRPECVVPE. NCQCANKRREKKACKEK \\
\hline $\begin{array}{l}\text { DmHR96 } \\
\text { Consensus }\end{array}$ & . \\
\hline BdE75 & . AHLETCEFTKEKVSANRCR \\
\hline BdE78 & .... ASVSVGAGGAGGMG \\
\hline BdHR3 & RACSLAAP ....... LSSVFLTCTPSS. . \\
\hline BdECR & . HPTCPLLPLCI VAKCRASNI PPL TRNCLLAVI YKLI VYCLGY \\
\hline BdHR96 & NSPTSI CS. . NI ELPSTSAG. . LLNPNSPTDLTSLATLFN. \\
\hline DmE75 & 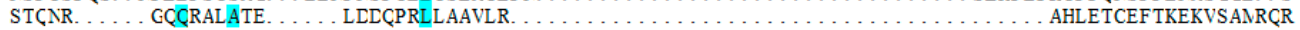 \\
\hline DmE78 & TSSTLHP SHL CQCCCCCHLLCCCCCCCHCPCLCCHHCL CCCPHVSGVRVKTPSTPCTPCMCSI ASSPSELGGCNS \\
\hline DmHR3 & 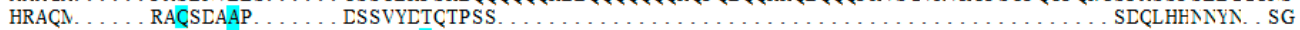 \\
\hline DmECR & $\ldots \ldots \ldots$ HATI PLLPLEI LAKCCARNI PSL TYNCLLAVI YKLI WYCLGY \\
\hline $\begin{array}{l}\text { DmHR96 } \\
\text { Consensus }\end{array}$ & HSPG. . . . . VGEQPSTSS. . . . . . SHPLPYI ANSPLF. \\
\hline BdE75 & 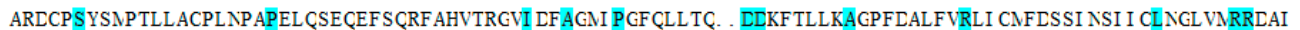 \\
\hline BdE78 & .... VGGGGGGVGG. NVGI TSVIN NGCGV.... \\
\hline BdHR3 & GYSSNEVSSPYGYGYSTSVTP. . . C CCTNAYLI SALYVLSTTYEPRGTI $\ldots \ldots$ LSLFVSHALGLI NLVLI KTLAEAHANTSTKLEAVHDNFFKSCD \\
\hline BdECR & ECPSLELLKRI TSTPLENESPNEVSFRHI TEI TI LTVQLI VEFAKGLPAFTKI PC. . ELCI TLLKACSSEVMLLRNARRYLHNSLSI FF AN NRSYTRLAY \\
\hline BdHR96 & LNSLESVL SEVI RI EFCAFN NLPEPRVKCECF Y YCTHNA ANC NSVCCCCCGYNGGHTCL CCPI I GPPTNAI NGRLL NEAEHNKLREL KL ASEAL YYPND \\
\hline DmE75 & RLCPSYSNPTLLACPL NPAPELCSECEF SCRF AHVI RGVI LFAGMI PGFCLLTC. . ELKFTLLKAGLFLALFVRLI CNFLSSI \\
\hline DmE78 & ANNNNNNN NSSSCNASGGSGVSVGVVVVGGHCCLVGGSN VGAGNGTLAHCQVGCHLGLAGT ANEL TVYLVI NCVSCAHRL NCSYTEEL TRELMRRPVT \\
\hline DmHR3 & YSN NEVGYGSPYGYSASVTP. . ...... CCTICYLI SALYVDSTTYEPRSTI I . . . DPEFI SHA \\
\hline $\mathrm{DmECR}$ & 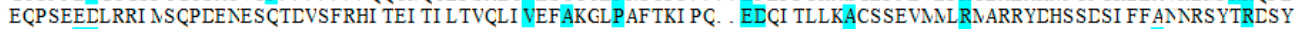 \\
\hline $\begin{array}{l}\text { DmHR96 } \\
\text { Consensus }\end{array}$ & I NSI ESVLSEVI RI EYCAFNSI CQAASRVKEENSYGT. . . . CSTYGGCNSAAN NSCPHLCQPI CAPST. CQLDRELNEAECQKLRELRLASEALYLPVD \\
\hline BdE75 & NGANARF. . LVLSTFNFAERINSANLSLAEI GLFCAI \\
\hline BdE78 & VPCNGI AT. . SVSETNEFCKI VL WCCYAGRVTPGVCRI VEF AKRVPGF CDF TCLLCLI LI KL GFFEVWL SH. .... \\
\hline BdHR3 & I SR........ I LYYKNL GCEEL VLECAEKL TCQI CNI I EF AKLI PGFNRL SCQDLCI LLLKT GSFELAI VR..... \\
\hline BdECR & KNAG. VAL. NI ELLLHFCRCQ YSNKVLNVEYALL TAI VI FS. LRPGLEKAELVEAI CNYYI NTLRVYI I NRHCGD \\
\hline BdHR96 & 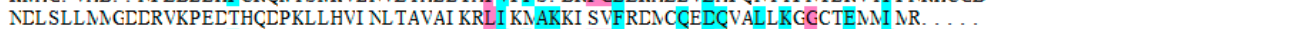 \\
\hline DmE75 & CNGANARF. . LVLSTFNFAERN NSNNL TCAEI GLFCAI VLI TPLRPGL RNLELI EKNYSRLKGCLCYI VACNRP. \\
\hline DmE78 & VPCNGI AS. . TVAESLEF CKI WL WCQCF S ARVTPGVCRI VEF AKRVPGFCDF TCDLCLI LI KL GFFEVWL TH. \\
\hline DmHR3 & VSR........ I LYYKNL GCEEEL WLLCAEKL TCQI CNI I EF AKLI PGFNRL SCQDLCQI LLLKTGSFELAI VR. ..... \\
\hline $\mathrm{DMECR}$ & KNAG. NAL. NI ELLLHFCRCMF SNKVLNVEYALL TAI VI FS. LRPGLEKACLLVEA CQSYYI LTLRI YI LNRHCGD \\
\hline DmHR96 & ELL SALN GLERI KPLETRHNPKLLCLI NLTAVAI KRLI KMAKKI TAFRLNCCELCVALLKGGCTEMM $N$ R. . . . \\
\hline
\end{tabular}

Figure 7. Conserved regions in the NR1 subfamily of $B$. dorsalis and D. melanogaster NR proteins. Amino acid sequences of the catalytic domains of the NR1 subfamily were aligned using DNAMAN 6 (https://www.lynnon.com/downloads.html). Identical and highly conserved amino acids are indicated by black (100\%), pink (75\%), and blue (50\%), respectively. 


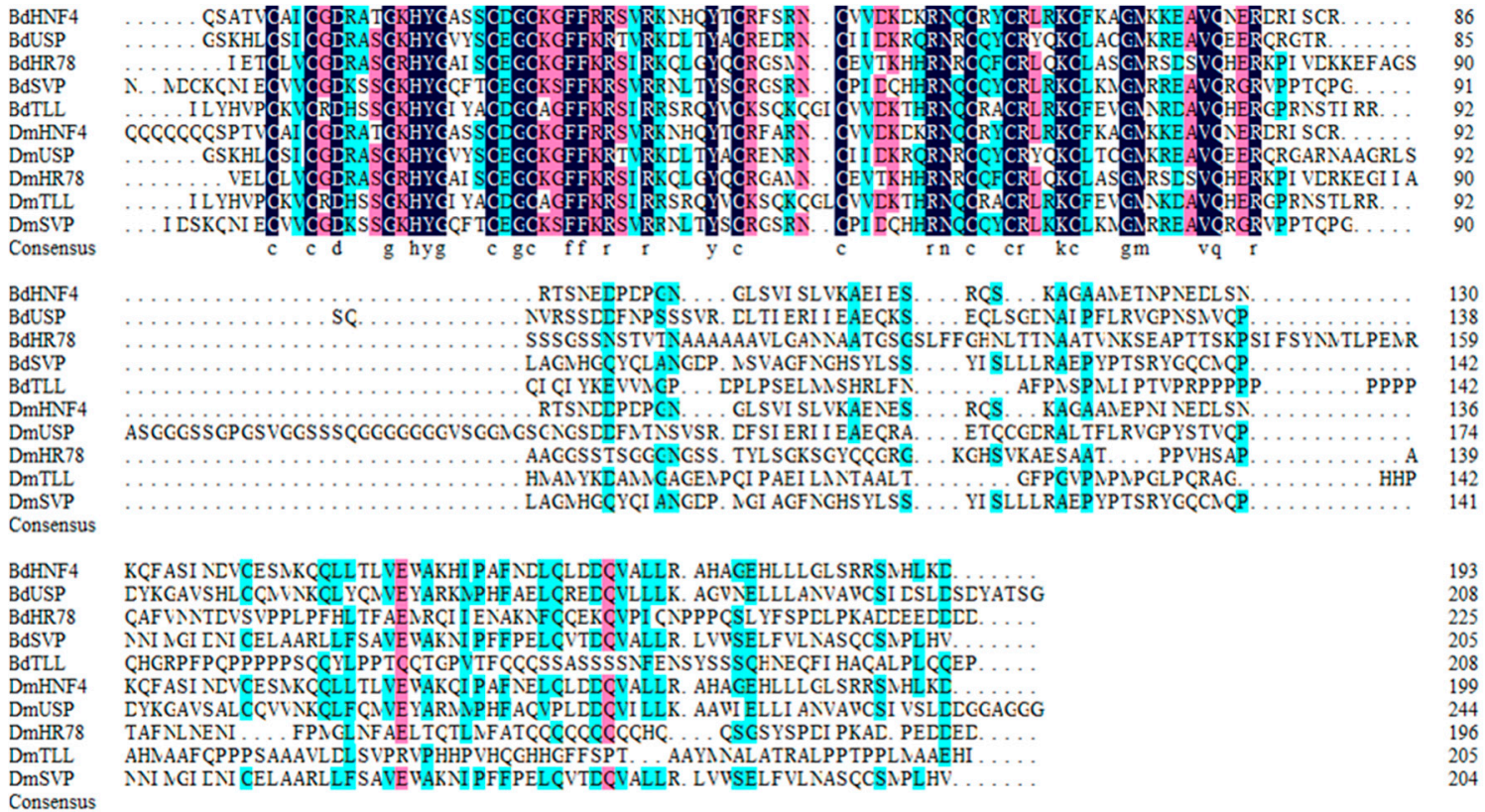

Figure 8. Conserved regions in the NR2 subfamily of NR proteins from B. dorsalis (excluding BdHR51, $B d D S F$, and BdHR83). Amino acid sequences of the catalytic domains of the NR2 subfamily were aligned using DNAMAN 6 (https://www.lynnon.com/downloads.html). Identical and highly conserved amino acids are indicated by black (100\%), pink (75\%), and blue (50\%), respectively.

\section{Discussion}

\subsection{NRO Subfamily}

All of the NR0 subfamily genes lacked LBDs, which is characteristic of the NR0A group [3]. The D. melanogaster KNRL has a 19 amino acid kni-box motif adjacent to the zinc fingers [19] and this motif was fully conserved in BdKNRL, BdKNI and BdEGON. In D. melanogaster, DmKNRL and $D m E G O N$ mediate the orchestration of embryogenesis and cell fate [20]. No NR0B members were detected among the BdNRs based upon similarity with the vertebrate NRs SHP and DAX1.

$D m K N I$ and DmKNRL were expressed in D. melanogaster at the blastocyst stage (Nauber et al., 1988) and a lack of DmKNI and DmKNRL expression led to head morphology and trachea development distortions in the late development stage [21]. However, when one or the other of the two genes underwent a single mutation, the head morphological defects were not observed, which indicated that they may have functional redundancy. DmEGON was expressed in four neurons and was briefly expressed in the embryonic gonad [22]. The time expression patterns of the NR0 subfamily of genes showed that $B d E G O N$ was highly expressed during $B$. dorsalis pupal development, which indicated it may be involved in the formation and differentiation of the adult nervous system.

\subsection{NR1 Subfamily}

One of these BdNRs belonged to the NR1D group and was named BdE75 because of its shared similarity with the D. melanogaster E75 receptor. Insect E75 represses the action of HR3 by binding nitric oxide as a ligand. Because of E75 can accommodate heme, it can bind small signaling molecules, such as carbon monoxide and nitric oxide, via the heme moiety [23]. Recently, microRNA let-7 was reported to target the E75 gene in the 20E signaling pathway in order to control larval-pupal development in B. dorsalis [24].

Insect HR3, a homolog of vertebrate vitamin A (retinoid-related orphan receptor), is a molting-regulated transcription factor that plays an important role in regulating the expression of tissue-specific genes involved in D. melanogaster molting and metamorphosis, and also is necessary for 
abdominal nerve cord formation [25]. From the phylogenetic analysis, it is uncertain whether the missing domains are true deletions or just artifacts. The qRT-PCR analysis showed that BdHR3 expression was significantly higher just prior to the larval-pupal molt stage and in five- and seven-day-old pupae than at any of the other stages. BdHR3 was highly expressed in the larval midgut and adult ovary, so we speculated that it may also be involved in larval digestion and adult sexual maturation.

E78 was the only member of the NR1E group, and its sequence was only 363 aa, which is shorter than those of the other members of NR1 (Table 1). The E78 sequence was short mainly because it lacked a DBD. In larval D. melanogaster, DmE78 expression was related to changes in molting hormone titer, because it was induced directly by a molting hormone and depended on 20E-induced protein synthesis to increase its expression level [26]. E78 also played a crucial role in proper egg production and for the maternal control of early embryogenesis. The functions of BdE78 are unclear but may include some of the functions of E78 in D. melanogaster.

Analysis of the $B$. dorsalis genome revealed the presence of an ecdysteroid receptor $(B d E c R)$ that belonged to the NR1H group (Table 1). Alignment analysis revealed that the deduced protein sequence shared an $86 \%$ identity with EcR-B1 of D. melanogaster, which indicated that this gene was highly conserved during dipteran evolution. Phylogenetic analysis revealed that $B d E c R$ was orthologous to the EcR proteins of other insect species. The qRT-PCR analysis showed that BdEcR was expressed at all tested developmental stages, and its expression reached obviously high levels just prior to the larval-pupal molt and in five-day-old pupae when compared with its expression at other stages. Moreover, the BdEcR gene was much more strongly expressed in the gut and Malpighian tubule than in the trachea and fat body, which indicated it may be involved in tissue-specific functions during larval development [27].

The $B$. dorsalis genome contained one NR with significant homology to D. melanogaster HR96. This receptor was in the NR1J group and was designated BdHR96. The homology between the BdHR96 and D. melanogaster HR96 sequences was not high, which indicated that gene differentiation may have occurred during dipteran evolution. From the phylogenetic analysis, it is uncertain whether the missing domains are true deletions or just artifacts. However, the function of BdHR96 is unclear. According to qPCR, high expression existed in the midgut of the adult and the Malpighian tubule of the larva. This indicated that HR96 may play roles in fat decomposition in the midgut. HR96 also was found to play an important role in the stress response of a variety of biomes [15].

\subsection{NR2 Subfamily}

The B. dorsalis genome contained a single member of the NR2A group that was designated BdHNF4 because of its similarity to D. melanogaster HNF4. In D. melanogaster, DmHNF4 plays an important role in maintaining glucose homeostasis [28]. Our qRT-PCR analysis showed that BdHNF4 was expressed at all tested developmental stages, except the egg stage, and was expressed in the fat body, Malpighian tubule, and midgut of the third instar larvae; however, its expression was not obvious in adults. The function of BdHNF4 may be somewhat similar to that of HNF4 in D. melanogaster.

Ultraspiracle (USP), a NR2B group member, was detected in the $B$. dorsalis genome and was designated BdUSP. In D. melanogaster, EcR and USP are induced by $20 \mathrm{E}$ and form heterodimers that initiate the molting cascade, and USP regulates (mainly adult) disc differentiation and midgut formation [29]. The high expression occurred in the midgut of the male and the ovary of the female, and also existed in the midgut and Malpighian tubule of the larvae (Figures 4 and 5). The function of BdUSP seems somewhat similar to that of USP in D. melanogaster. In addition, some influence on ovarian development may also exist.

The $B$. dorsalis genome contained a single member of the NR2D group. We designated it BdHR78 (because it was most closely related to D. melanogaster HR78) and C. capitata HR78, both of which belong to the NR2D group. HR78 was found to be involved in regulating the expression of genes in the ecdysone and Notch signaling pathways [16]. The functions of $B d H R 78$ have not been reported; 
however, our qRT-PCR analysis indicated that BdHNF4 and BdHR78 were expressed in the midgut of third instar larvae, which indicated that these two genes may have some similar functions (Figure 5).

The $B$. dorsalis genome contained four members of the NR2E group (Table 1). Homologs of BdHR83 and BdDSF could not be verified because their sequences were incomplete. TLL in Drosophila melanogaster played an important role in visual, nervous, and digestive system development [30]. $B d T L L$ was significantly highly expressed in the embryo stage and in the ovaries of seven- to nine-day-old females, which indicated it may be involved in the sexual maturation of the ovaries, but this needs to be verified (Figs. 3-5). Notably, although BdHR51 was highly expressed in the larval central nervous system (Figure 5), its function is still unknown; however, it has been reported to regulate fly wing development and reproduction [31].

The B. dorsalis genome contained a single member of the NR2F group. It was designed as BdSVP because of its homology to SVP in D. melanogaster. DmSVP can form an allodimer with USP and compete with EcR-USP for DNA binding sites. SVP plays an important role in neural development and is involved in the establishment of the central nervous system of D. melanogaster embryos [32], as well as in the proliferation and differentiation of different neuroblasts in later developmental stages [33]. The specific regulatory of SVP indicated that it may repress the induction of the FMRFamide gene prior to target contact [34].

\subsection{NR3 Subfamily}

The B. dorsalis genome contained only one member of the NR3B group (Table 1, Figure 1). It was designated as $B d E R R$. Structurally, $B d E R R$ was similar to the estrogen-related receptors (ERRs) of mammals, which indicated that ERRs have highly conserved structural characteristics. A very recent study suggested that the $D m E c R$ and $D m E R R$ jointly regulated the expression of glucose metabolism-related genes [35], but their specific functions in B. dorsalis are not yet clear. Our qRT-PCR analysis showed that $B d E R R$ was expressed in all development stages, but no obviously high expression was detected (Figure 3).

\subsection{NR4 Subfamily}

The B. dorsalis genome contained only one member of the NR4A group (Table 1, Figure 1). It was designated $B d H R 38$. HR38 may be a true orphan receptor, because no ligand binding pocket or coactivator-binding site was found in its 3D structure [36]. In D. melanogaster, HR38 was highly expressed in the gut and integument of larva, and its transcript levels fluctuated according to their nutritional status [37]. Our qRT-PCR analysis indicated that BdHR38 was highly expressed in the central nervous system of the third instar larva but not in the cuticle (Figure 5). At present, we are unable to explain this result.

\subsection{NR5 Subfamily}

The B. dorsalis genome contained two members of the NR5 subfamily: BdFTZ-F1, a member of the NR5A group and BdHR39, a member of the NR5B group (Table 1, Figure 1). Phylogenetic analysis indicated that BdFTZ-F1 was orthologous to the FTZ-F1 proteins of D. melanogaster (Figure 2). Acetylation of FTZ-F1 and histone H4K5 was required for the fine-tuning of ecdysone biosynthesis during Drosophila metamorphosis [38]. Our qRT-PCR analysis indicated that FTZ-F1 was not highly expressed during pupation, but showed obvious expression in M A9 (Figure 3). This indicated that FTZ-F1 may have a different function in B. dorsalis compared to that of D. melanogaster.

HR39 was the only member of the NR5B group. It shared a high sequence similarity with FTZ-F1, and both subtypes were expressed in the later stages of D. melanogaster growth and development [39]. Our qRT-PCR analysis indicated that itwas expressed obviouslyin the Malpighian tubuleand midgut at larval stage. However, the specific function of HR39 in B.dorsalis is not clear, nor is it supported by citation. 


\subsection{NR6 Subfamily}

The B. dorsalis genome contained one member of the NR6A group (Table 1, Figure 1). From the phylogenetic analysis, it is uncertain whether the missing domains are true deletions or just artifacts. HR4 was found to be regulated by ecdysteroids and, thus, may coordinate the molting process [39]. In $D$. melanogaster, HR4 functions as a repressor or an inducer when mediating growth and maturation [40].

\section{Conclusions}

So far, 21 NR genes have been identified in B. dorsalis. Nineteen of these genes were successfully amplified; the exceptions were HR83 and DSF. Phylogenetic analysis showed that members of the BdNR family were highly homologous to DmNR family members. The NR0 subfamily members (BdKNRL, BdEGON, and BdKNI) all lacked LBDs, and BdHR83 in the NR2 subfamily also lacked an LBD. The other members of the NR2 subfamily (BdHR51 and BdDSF) and BdE78 in the NR1 subfamily all lacked DBDs. The absence of these domains may lead to functional changes, but the effects of this on specific functional regulatory mechanisms are still not fully understood.

NR proteins have ubiquitous essential functions in regulating many aspects of metazoan physiology. The biological functions of some of the NRs have been established and related ligands have been identified [41]. However, the functions of many of the NRs remain elusive. The identification of NR genes in $B$. dorsalis is the first step to gain more insight into the evolutionary and structural aspects of these important transcription factors. Spatiotemporal transcript profiles provide a foundation for identifying the physiological functions of specific NRs.

Supplementary Materials: The following are available online at http://www.mdpi.com/2075-4450/11/2/126/s1, Table S1: Sequences from Drosophila melanogaster that were used to identify NR families in Bactrocera dorsalis, Table S2: Sequences of 90 nuclear receptor (NR) proteins from five insect species for the phylogenetic analysis, Table S3: List of specific primers for qRT-PCR NR, Table S4: The mean, SE and significances of developmental mRNA expression profiles of 19 B. dorsalis NR genes (excluding BdHR83 and BdDSF), Table S5: The mean, SE and significances of tissue-specific expression of 19 B. dorsalis NR genes (excluding BdHR83 and BdDSF) from 4-day-old adults, Table S6: The mean, SE and significances of tissue-specific expression of 19 B. dorsalis NR genes (excluding $B d H R 83$ and BdDSF) from third instar larvae.

Author Contributions: P.-J.Y., W.D. and E.-H.C. designed the research. P.-J.Y., W.H. and Z.-H.S. performed the experiments. The research was resourced by J.-J.W. and W.D.; P.-J.Y., E.-H.C and S.-H.L. analyzed the data. P.-J.Y., W.D. and S.-H.L. wrote the manuscript. All authors have read and agreed to the published version of the manuscript.

Funding: This work was supported by the National Natural Science Foundation of China $(31872031,31672030)$, Chongqing Research Program of Basic Research and Frontier Technology (cstc2018jcyjAX0285), the Fundamental Research Funds for the Central Universities (XDJK2019AC004) of China, and the earmarked fund for the Modern Agroindustry (Citrus) Technology Research System of China (CARS-27).

Acknowledgments: We thank W.L. and Y.Y., Innovation Research Team of Insect Molecular Ecology, Academy of Agriculture Science, Southwest University, for providing the source of the insects and giving advice on this research. We also appreciate editors and reviewers for their professional suggestions and patience.

Conflicts of Interest: The authors declare no conflict of interest.

\section{References}

1. Evans, R.M. The nuclear receptor superfamily: A Rosetta Stone for physiology. Mol. Endocrinol. 2005, 19, 1429-1438. [CrossRef] [PubMed]

2. Benoit, G.; Cooney, A.; Giguere, V.; Ingraham, H.; Lazar, M.; Muscat, G.; Perlmann, T.; Renaud, J.P.; Schwabe, J.; Sladek, F.; et al. International Union of Pharmacology. LXVI. Orphan nuclear receptors. Pharmacol. Rev. 2006, 58, 798-836. [CrossRef] [PubMed]

3. Duarte, J.; Perriere, G.; Laudet, V.; Robinson-Rechavi, M. Nurebase: Database of nuclear hormone receptors. Nucleic Acids Res. 2002, 30, 364-368. [CrossRef] [PubMed]

4. Germain, P.; Staels, B.; Dacquet, C.; Spedding, M.; Laudet, V. Overview of nomenclature of nuclear receptors. Pharmacol. Rev. 2006, 58, 685-704. [CrossRef] [PubMed] 
5. Robinson-Rechavi, M.; Carpentier, A.S.; Duffraisse, M.; Laudet, V. How many nuclear hormone receptors are there in the human genome? Trends Genet. 2001, 17, 554-556. [CrossRef]

6. Sluder, A.E.; Maina, C.V. Nuclear receptors in nematodes: Themes and variations. Trends Genet. 2001, 17, 206-213. [CrossRef]

7. Zhang, Z.D.; Burch, P.E.; Cooney, A.J.; Lanz, R.B.; Pereira, F.A.; Wu, J.Q.; Gibbs, R.A.; Weinstock, G.; Wheeler, D.A. Genomic analysis of the nuclear receptor family: New insights into structure, regulation, and evolution from the rat genome. Genome Res. 2004, 14, 580-590. [CrossRef]

8. King-Jones, K.; Thummel, C.S. Nuclear receptors-A perspective from Drosophila. Nat. Rev. Genet. 2005, 6, 311-323. [CrossRef]

9. Cheng, D.; Xia, Q.; Duan, J.; Wei, L.; Huang, C.; Li, Z.; Wang, G.; Xiang, Z. Nuclear receptors in Bombyx mori: Insights into genomic structure and developmental expression. Insect Biochem. Mol. Biol. 2008, 38, 1130-1137. [CrossRef]

10. Velarde, R.A.; Robinson, G.E.; Fahrbach, S.E. Nuclear receptors of the honey bee: Annotation and expression in the adult brain. Insect Mol. Biol. 2006, 15, 583-595. [CrossRef]

11. Bonneton, F.; Chaumot, A.; Laudet, V. Annotation of Tribolium nuclear receptors reveals an increase in evolutionary rate of a network controlling the ecdysone cascade. Insect Biochem. Mol. Biol. 2008, 38, 416-429. [CrossRef] [PubMed]

12. Jarvela, A.M.C.; Pick, L. The Function and Evolution of Nuclear Receptors in Insect Embryonic Development. In Nuclear Receptors in Development and Disease; Forrest, D., Tsai, S., Eds.; Elsevier Academic Press Inc.: San Diego, CA, USA, 2017; Volume 125, pp. 39-70.

13. Bernardo, T.J.; Dubrovskaya, V.A.; Jannat, H.; Maughan, B.; Dubrovsky, E.B. Hormonal Regulation of the E75 Gene in Drosophila: Identifying Functional Regulatory Elements through Computational and Biological Analysis. J. Mol. Biology 2009, 387, 794-808. [CrossRef] [PubMed]

14. Mazina, M.Y.; Kocheryzhkina, E.V.; Nikolenko, J.V.; Krasnov, A.N.; Georgieva, S.G.; Vorobyeva, N.E. Nuclear receptors EcR, Usp, E75, DHR3, and ERR regulate transcription of ecdysone cascade genes. Dokl. Biochem. Biophys. 2017, 473, 145-147. [CrossRef] [PubMed]

15. Afschar, S.; Toivonen, J.M.; Hoffmann, J.M.; Tain, L.S.; Wieser, D.; Finlayson, A.J.; Driege, Y.; Alic, N.; Emran, S.; Stinn, J.; et al. Nuclear hormone receptor DHR96 mediates the resistance to xenobiotics but not the increased lifespan of insulin-mutant Drosophila. Proc. Natl. Acad. Sci. USA 2016, 113, 1321-1326. [CrossRef] [PubMed]

16. Marxreiter, S.; Thummel, C.S. Adult functions for the Drosophila DHR78 nuclear receptor. Dev. Dyn. 2018, 247, 315-322. [CrossRef]

17. Clarke, A.R.; Armstrong, K.F.; Carmichael, A.E.; Milne, J.R.; Raghu, S.; Roderick, G.K.; Yeates, D.K. Invasive phytophagous pests arising through a recent tropical evolutionary radiation: The Bactrocera dorsalis complex of fruit flies. Annu. Rev. Entomol. 2005, 50, 293-319. [CrossRef]

18. Wang, J.J.; Wei, D.; Dou, W.; Hu, F.; Liu, W.F.; Wang, J.J. Toxicities and synergistic effects of several insecticides against the oriental fruit fly (Diptera: Tephritidae). J. Econ. Entomol. 2013, 106, 970-978. [CrossRef]

19. Rothe, M.; Nauber, U.; Jackle, H. Three hormone receptor-like Drosophila genes encode an identical DNA-binding finger. Embo J. 1989, 8, 3087-3094. [CrossRef]

20. Lundell, M.J.; Hirsh, J. Eagle is required for the specification of serotonin neurons and other neuroblast 7-3 progeny in the Drosophila CNS. Development 1998, 125, 463-472.

21. Chen, C.K.; Kuhnlein, R.P.; Eulenberg, K.G.; Vincent, S.; Affolter, M.; Schuh, R. The transcription factors Knirps and Knirps related control cell migration and branch morphogenesis during Drosophila tracheal development. Development 1998, 125, 4959-4968.

22. Higashijima, S.; Shishido, E.; Matsuzaki, M.; Saigo, K. Eagle, a member of the steroid receptor gene superfamily, is expressed in a subset of neuroblasts and regulates the fate of their putative progeny in the Drosophila CNS. Development 1996, 122, 527-536. [PubMed]

23. Marvin, K.A.; Reinking, J.L.; Lee, A.J.; Pardee, K.; Krause, H.M.; Burstyn, J.N. Nuclear receptors Homo sapiens Rev-erb beta and Drosophila melanogaster E75 are thiolate-ligated heme proteins which undergo redox-mediated ligand switching and bind CO and NO. Biochemistry 2009, 48, 7056-7071. [CrossRef] [PubMed] 
24. Peng, W.; Zheng, W.W.; Tariq, K.; Yu, S.N.; Zhang, H.Y. MicroRNA Let-7 targets the ecdysone signaling pathway E75 gene to control larval-pupal development in Bactrocera dorsalis. Insect Sci. 2019, 26, 229-239. [CrossRef] [PubMed]

25. Ruaud, A.-F.; Lam, G.; Thummel, C.S. The Drosophila nuclear receptors DHR3 and beta FTZ-F1 control overlapping developmental responses in late embryos. Development 2010, 137, 123-131. [CrossRef]

26. Ables, E.T.; Bois, K.E.; Garcia, C.A.; Drummond-Barbosa, D. Ecdysone response gene E78 controls ovarian germline stem cell niche formation and follicle survival in Drosophila. Dev. Biology 2015, 400, 33-42. [CrossRef]

27. Cong, L.; Yang, W.J.; Shen, G.M.; Dou, W.; Wang, J.J. Molecular characterization of the cDNA encoding ecdysone receptor isoform B1 and its expression in the oriental fruit fly, Bactrocera dorsalis (Diptera: Tephritidae). Fla. Entomol. 2012, 95, 650-658. [CrossRef]

28. Barry, W.E.; Thummel, C.S. The Drosophila HNF4 nuclear receptor promotes glucose-stimulated insulin secretion and mitochondrial function in adults. Elife 2016, 5, e11183. [CrossRef]

29. Hall, B.L.; Thummel, C.S. The RXR homolog Ultraspiracle is an essential component of the Drosophila ecdysone receptor. Development 1998, 125, 4709-4717.

30. Janssens, H.; Crombach, A.; Wotton, K.R.; Cicin-Sain, D.; Surkova, S.; Lim, C.L.; Samsonova, M.; Akam, M.; Jaeger, J. Lack of tailless leads to an increase in expression variability in Drosophila embryos. Dev. Biol. 2013, 377, 305-317. [CrossRef]

31. Sung, C.; Wong, L.E.; Sen, L.Q.C.; Nguyen, E.; Lazaga, N.; Ganzer, G.; McNabb, S.L.; Robinow, S. The unfulfilled/DHR51 gene of Drosophila melanogaster modulates wing expansion and fertility. Dev. Dyn. 2009, 238, 171-182. [CrossRef]

32. Kanai, M.I.; Okabe, M.; Hiromi, Y. Seven-up controls switching of transcription factors that specify temporal identities of Drosophila neuroblasts. Dev. Cell 2005, 8, 203-213. [CrossRef]

33. Maurange, C.; Cheng, L.; Gould, A.P. Temporal transcription factors and their targets schedule the end of neural proliferation in Drosophila. Cell 2008, 133, 891-902. [CrossRef] [PubMed]

34. Berndt, A.J.E.; Tang, J.C.Y.; Ridyard, M.S.; Lian, T.S.; Keatings, K.; Allan, D.W. Gene regulatory mechanisms underlying the spatial and temporal regulation of target-dependent gene expression in Drosophila neurons. PLoS Genet. 2015, 11. [CrossRef] [PubMed]

35. Kovalenko, E.V.; Mazina, M.Y.; Krasnov, A.N.; Vorobyeva, N.E. The Drosophila nuclear receptors ECR and ERR jointly regulate the expression of genes involved in carbohydrate metabolism. Insect Biochem. Mol. Biol. 2019, 112, 103184. [CrossRef] [PubMed]

36. Baker, K.D.; Shewchuk, L.M.; Kozlova, T.; Makishima, M.; Hassell, A.; Wisely, B.; Caravella, J.A.; Lambert, M.H.; Reinking, J.L.; Krause, H.; et al. The Drosophila orphan nuclear receptor DHR38 mediates an atypical ecdysteroid signaling pathway. Cell 2003, 113, 731-742. [CrossRef]

37. Ruaud, A.-F.; Lam, G.; Thummel, C.S. The Drosophila NR4A nuclear receptor DHR38 regulates carbohydrate metabolism and glycogen storage. Mol. Endocrinol. 2011, 25, 83-91. [CrossRef] [PubMed]

38. Borsos, B.N.; Pankotai, T.; Kovacs, D.; Popescu, C.; Pahi, Z.; Boros, I.M. Acetylations of FTZ-F1 and histone H4K5 are required for the fine-tuning of ecdysone biosynthesis during Drosophila metamorphosis. Dev. Biol. 2015, 404, 80-87. [CrossRef]

39. Sullivan, A.A.; Thummel, C.S. Temporal profiles of nuclear receptor gene expression reveal coordinate transcriptional responses during Drosophila development. Mol. Endocrinol. 2003, 17, 2125-2137. [CrossRef]

40. Ou, Q.; Magico, A.; King-Jones, K. Nuclear receptor DHR4 controls the timing of steroid hormone pulses during Drosophila development. PLoS Biol. 2011, 9, e1001160. [CrossRef]

41. Thomson, S.A.; Baldwin, W.S.; Wang, Y.H.; Kwon, G.; LeBlanc, G.A. Annotation, phylogenetics, and expression of the nuclear receptors in Daphnia pulex. BMC Genom. 2009, 10, 500. [CrossRef]

(C) 2020 by the authors. Licensee MDPI, Basel, Switzerland. This article is an open access article distributed under the terms and conditions of the Creative Commons Attribution (CC BY) license (http://creativecommons.org/licenses/by/4.0/). 